\title{
Hyperspectral optical near-field imaging: Looking graded photonic crystals and photonic metamaterials in color
}

Cite as: Appl. Phys. Lett. 101, 141108 (2012); https://doi.org/10.1063/1.4756902

Submitted: 19 June 2012 - Accepted: 17 September 2012 • Published Online: 02 October 2012

Jean Dellinger, K. Van Do, Xavier Le Roux, et al.

ARTICLES YOU MAY BE INTERESTED IN

Self-focusing media using graded photonic crystals: Focusing, Fourier transforming and imaging, directive emission, and directional cloaking

Journal of Applied Physics 110, 053103 (2011); https://doi.org/10.1063/1.3630116

Graded photonic crystals curve the flow of light: An experimental demonstration by the mirage effect

Applied Physics Letters 92, 133501 (2008); https://doi.org/10.1063/1.2901684

A hyperspectral imaging system for an accurate prediction of the above-ground biomass of individual rice plants

Review of Scientific Instruments 84, 095107 (2013); https://doi.org/10.1063/1.4818918

耳QBLOX

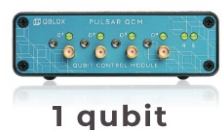

Shorten Setup Time Auto-Calibration More Qubits

Fully-integrated Quantum Control Stacks Ultrastable DC to $18.5 \mathrm{GHz}$ Synchronized $<<1$ ns Ultralow noise

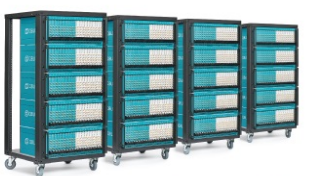

100s qubits

visit our website > 


\title{
Hyperspectral optical near-field imaging: Looking graded photonic crystals and photonic metamaterials in color
}

\author{
Jean Dellinger, ${ }^{1} \mathrm{~K}$. Van Do, ${ }^{2}$ Xavier Le Roux, ${ }^{2}$ Frédérique de Fornel, ${ }^{1}$ Eric Cassan, ${ }^{2, a)}$ \\ and Benoît Cluzel ${ }^{1, a)}$ \\ ${ }^{1}$ Groupe d'Optique de Champ Proche-Laboratoire Interdisciplinaire Carnot de Bourgogne, UMR CNRS 6303, \\ Université de Bourgogne, Dijon, France \\ ${ }^{2}$ Institut d'Electronique Fondamentale, Université Paris-Sud, CNRS, 91405 Orsay, France
}

(Received 19 June 2012; accepted 17 September 2012; published online 2 October 2012)

\begin{abstract}
Using a scanning near-field optical microscope operating with a hyperspectral detection scheme, we report the direct observation of the mirage effect within an on-chip integrated artificial material made of a two dimensional graded photonic crystal. The light rainbow due to the material dispersion is quantified experimentally and quantitatively compared to three dimensional plane wave assisted Hamiltonian optics predictions of light propagation. (C) 2012 American Institute of Physics. [http://dx.doi.org/10.1063/1.4756902]
\end{abstract}

Artificial materials at optical frequencies have raised a strong interest in the last years, including photonic metamaterials, ${ }^{1}$ graded photonic crystals, ${ }^{2-4}$ and simple gradient index structures. ${ }^{5}$ The main common objective of these approaches is achieving a tight control of the electromagnetic wave fields to play with light properties and thus propose versatile optical properties over the space and the spectrum. For instance, recent works have focused on the possible re-use of space, ${ }^{6}$ invisibility cloaks, ${ }^{7}$ and devices mimicking celestial mechanics. ${ }^{8}$

To provide in situ observations of the electromagnetic fields behavior inside these artificial structures and thus to improve our understanding of the involved light matter interactions, the scanning near-field optical microscopy (SNOM) techniques have raised an increasing interest over this last decade. Their spatial resolution being far beyond the diffraction limit ${ }^{9}$ are able to probe the electromagnetic (EM) fields at the same length scale of the artificial patterning as well as to detect the untravelling waves in the far-field, ${ }^{10}$ namely, the evanescent and leaky waves. The recent works in this field have reported different detection schemes and nearfield probes morphologies allowing the observation of the electric $^{11,12}$ and magnetic ${ }^{13}$ components of the EM fields and revealing their complex amplitudes ${ }^{14}$ or their intensities as well as their temporal dynamics. ${ }^{15,16}$ However, all these approaches suffer from a short spectral range, limited by the frequency shifters bandwidth in the interferomeric schemes and by the availability of commercial laser sources in any case. As discussed earlier, the versatile properties of the artificial materials over the spectrum would also require a broadband near-field detection scheme able to quantify the dispersion of the EM fields inside them. This issue is addressed here by developing a hyperspectral scanning nearfield optical microscope (Hyp-SNOM). Using a broadband illumination and detection as proposed at visible wavelengths in plasmonics, ${ }^{17}$ the Hyp-SNOM used here enables

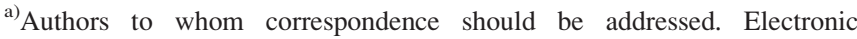
addresses: eric.cassan@u-psud.fr and benoit.cluzel@u-bourgogne.fr.
}

the direct observation of light dispersion within an on-chip integrated graded photonic crystal operating at near-infrared wavelengths. To provide an overview of the capabilities of the near-field technique reported here, the fascinating mirage effect $^{4,18}$ is directly visualized in the optical near-field of a two dimensional graded photonic crystal (GPhC). The spectral and spatial dispersions of curved light beams are then quantified experimentally and compared quantitatively to three dimensional plane wave assisted Hamiltonian optics ${ }^{2}$ (HO) predictions of light propagation.

The GPhC considered in this work operates in the diffraction regime, i.e., close to the photonic crystal $(\mathrm{PhC})$ bandgap. The light propagation is governed by the local $\mathrm{PhC}$ band structure and not only by a simple index map, thus allowing the exploitation of $\mathrm{PhC}$ dispersive effects. The underlying idea is thus to adjust "on-the-fly," not the local index of the optical material, but the local curvature of the equi-frequency surface (EFS) governing the light group velocity direction within the GPhC area. ${ }^{19,20}$ Figure 1 shows an overview of the considered structure, consisting in a square shape $\mathrm{GPhC}$ area made of a $45^{\circ}$-rotated square lattice photonic crystal lattice with a gradual filling factor. The design of the structure was based on the use of a $260 \mathrm{~nm}$ thick SOI wafer with a $2 \mu \mathrm{m}$ of buried oxide layer and a $a=380 \mathrm{~nm}$ lattice period. ${ }^{19}$ Figure 1 also shows the electric field intensity calculated at normalized frequency $\omega=a / \lambda=0.25$ $(\lambda=1520 \mathrm{~nm})$ using FDTD simulation in transverse electric (TE) light polarization. As seen in Figure 1, light bending occurs towards the region with the highest filling factor, namely, the lowest long range spatially averaged refractive index, evidencing that the structure principle does not rely on a gradual index effect. Figure 1(b) shows the corresponding fabricated structure using electron-beam lithography and $\mathrm{SF}_{6} / \mathrm{O}_{2}$ anisotropic etching process and carefully accounting the proximity effects.

An overview of the Hyp-SNOM experimental setup is presented in Figure 2. A supercontinuum laser source with an output power up to $0.1 \mathrm{~mW} / \mathrm{nm}$ is used as an intense broadband excitation over the $400 \mathrm{~nm}-1.7 \mu \mathrm{m}$ spectral range. 

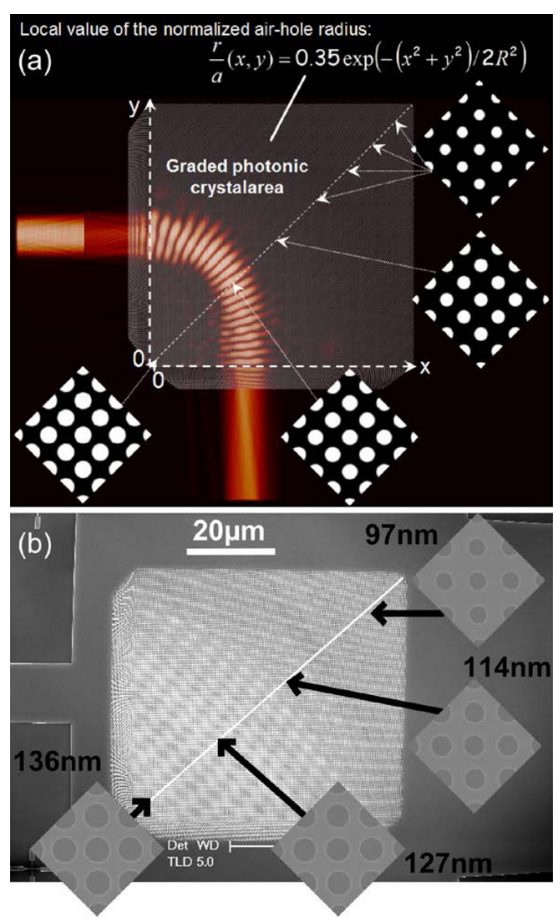

FIG. 1. (a) Overview of the GPhC area showing the simulated field at normalized frequency $\omega=\mathrm{a} / \lambda=0.25$ using FDTD simulation (air holes are etched into a SOI wafer). (b) SEM view of the fabricated GPhC structure with detailed views of some parts.

The evanescent decay of the light guided inside the material of interest is collected by a near-field probe made of a tapered monomode silica fiber at $4 \mathrm{~nm}$ above the surface. A free space spectrometer is then used to disperse the collected light, and the spectra are recorded synchronously with the probe scan every $50 \mathrm{~ms}$ on a cooled InGaAs camera for nearinfrared wavelengths or on an intensified charge coupled device (iCCD) for visible operations. In this configuration, a hyperspectral matrix as shown in Figure 2 is obtained in few tenths of minutes, the total acquisition time being defined by the detected intensity level, the desired spatial and spectral resolutions, and the near-field probe scan rate.

Using this setup, we directly measured the light propagation and dispersion inside the $\mathrm{GPhC}$ for a TE polarization. For silicon based materials, only near-infrared wavelengths above the Si electronic bandgap propagate inside the SOI chip, and the near-field detection is thus restricted to the 1200-1650 nm spectral range. As a result, the hyperspectral matrix shown in Figure 2 is recorded in 30 minutes with a $290 \mathrm{~nm}$ spatial resolution over a $45 \times 90 \mu \mathrm{m}$ image and with a $1 \mathrm{~nm}$ spectral resolution over the total spectral range. Then different representations of the matrix permit to emphasize the spatial or the spectral properties of the GPhC.

A conventional approach to project the hyperspectral matrix is the representation of the EM field maps at selected wavelengths. As shown in Figures 3(b)-3(e), this provides for the first time the direct experimental proof of the mirage effect due to the gradual nature of the PhC. Using this representation, a movie available online as a supporting media file shows the dynamical evolution of the EM field maps over the whole spectral range. As visible in the media but not presented in the figure, the wavelengths shorter than

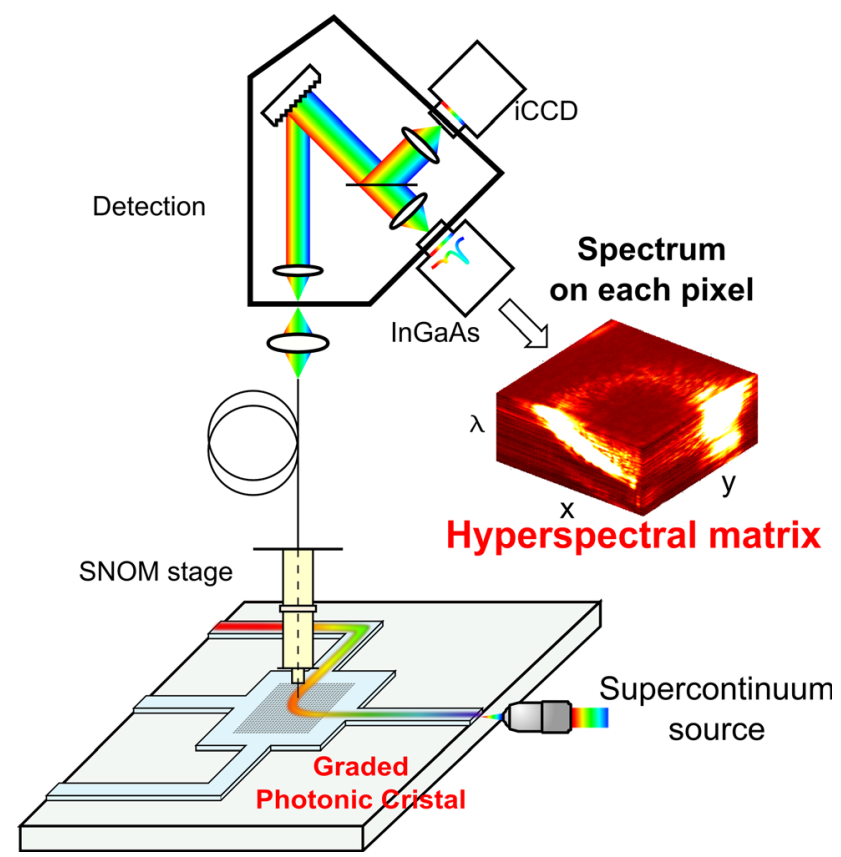

FIG. 2. Overview of the Hyp-SNOM experimental setup. During the scan controlled by the SNOM stage, we record on each pixel, the spectrum of the light collected by the near-field probe. This setup provides a 3D hyperspectral matrix over a broad spectral range.

$1400 \mathrm{~nm}$ do not penetrate in the $\mathrm{PhC}$ area and are reflected by the TE photonic bandgap of the GPhC. Then, the mirage effect takes place at upper wavelengths from $1400 \mathrm{~nm}$ to $1650 \mathrm{~nm}$. In this range, the chromatic dispersion of this effect is clearly evidenced by the progressive penetration of the light beam within the $\mathrm{PhC}$ area and the displacement of
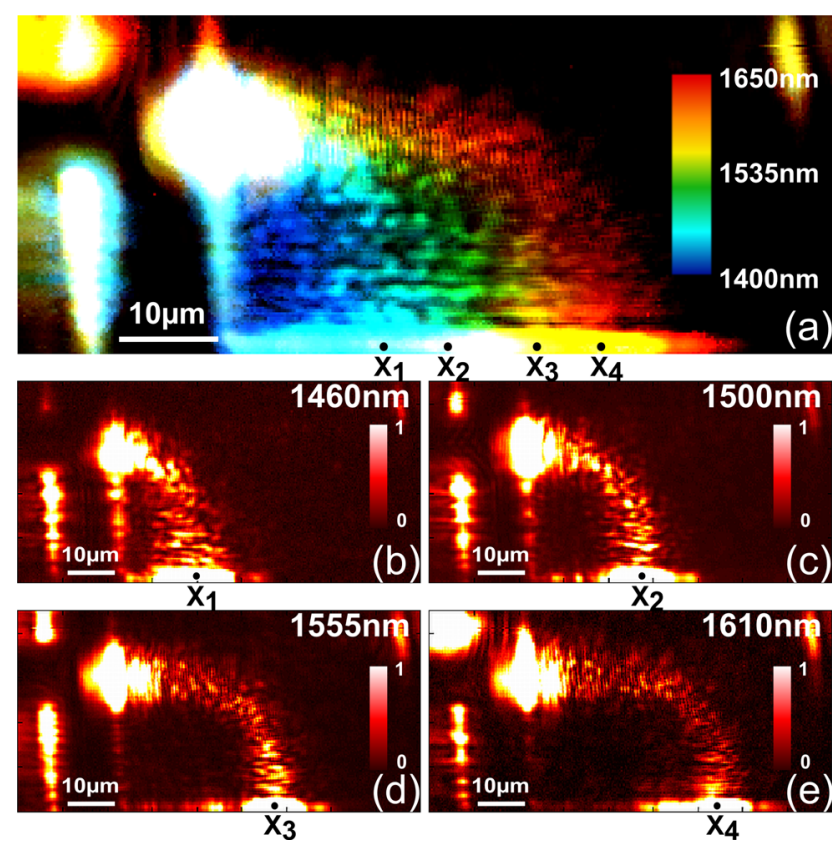

FIG. 3. Hyp-SNOM images: (a) "white" hyperspectral image which provides an overall knowledge of the structure behaviour. (b)-(e) EM field maps obtained from hyperspectral movie, for a TE light polarization at $\lambda=1460,1500,1555,1610 \mathrm{~nm}$, respectively (enhanced online) [URL: http://dx.doi.org/10.1063/1.4756902.1]. 
(a)

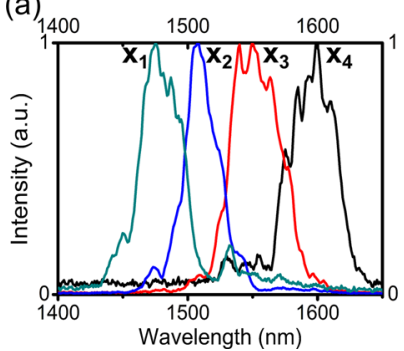

(b)

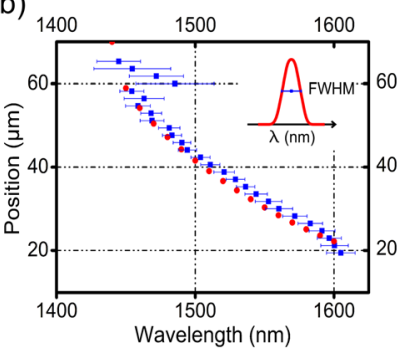

(c)

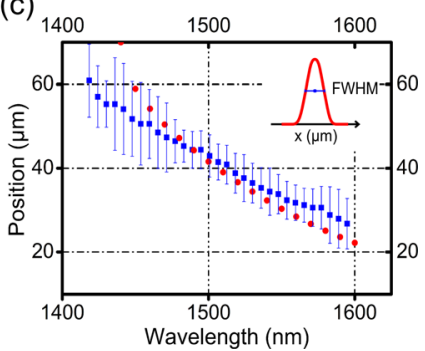

FIG. 4. Quantitative estimation of the wavelength dispersion properties of the fabricated GPhC using Hyp-SNOM measurements: (a) Spectral profiles recorded at the different coordinates depicted in Figs. 3(b)-3(e). Position of the centre of the output optical beam along the X-axis shown in Fig. 1 as a function of light wavelength: filled blue squares correspond to Hyp-SNOM measurements (b) with horizontal segments showing the beam FWHM spectral size, (c) with vertical segments showing the beam FWHM spatial size, while red circles correspond to Hamiltonian optics calculations based on the GPhC band structure extraction after 3D-PWE calculations.

its curvature while increasing the wavelength. Another feature clearly visible here is the absence of spatial beam spreading over a main part of the spectrum, providing a direct proof of the light beam collimation all along the curved trajectories.

Then, mimicking the classical representation of light dispersion through a glass prism, ${ }^{21}$ the hyperspectral matrix can be plotted on a single "white" image by using a colorcoded scale over the spectral range where the mirage effect takes place (Figure 3(a)). As a result, this representation provides on a single image of the light rainbow due to the artificial material dispersion.

To quantitatively estimate the dispersion of the GPhC studied here, we directly resort to the analysis of the hyperspectral matrix. All along the output interface of the GPhC area (namely, along the $\mathrm{x}$-axis in the figures), we extracted the optical spectra. Four snapshots of the spectra at different $\mathrm{x}$, corresponding to the $\times 1$ to $\times 4$ coordinates in Figure 3, are plotted in Figure 4(a). Each spectrum was then fitted by a Gaussian function to estimate its central wavelength as well as its spectral full width at half maximum (FWHM). Repeating this protocol all along the $\mathrm{x}$-axis permits to extract the dispersion relationships plotted in Figures 4(b) and 4(c), which show the light beam position along the output interface in respect to the excitation wavelength. For each position, the spectral (Figure 4(b)) and spatial (Figure 4(c)) FWHMs of the light beam are superimposed by horizontal and vertical segments. As a result, it is found that the output beam shifts almost linearly with a slope of $0.25 \mu \mathrm{m} / \mathrm{nm}$ in the $1470-1600 \mathrm{~nm}$ spectral range without noticeable spatial or spectral spreading. This dispersion value is comparable to the one achievable, thanks to the $\mathrm{PhC}$ superprism effect ${ }^{22}$ over almost the same propagation length about $0.4 \mu \mathrm{m} / \mathrm{nm}$, but the strong beam spreading inherent to the superprism effect appears here drastically reduced.

To compare these experimental results to the numerical predictions, we resort to the band diagram analysis of the GPhC. The EFSs of the GPhC considered here correspond to a region close to the edge of the first TE-like band near the bandgap between band 1 and band 2 and can be described by an analytical relationship depending on two parameters depending on the local filling factor and the light normalized frequency, respectively. ${ }^{19}$ Using this approach, we extracted these two parameters from 3D plane wave expansion (PWE) simulations, taking into account the asymmetry of the SOI wafer (air top cladding). Then, using the equations of $\mathrm{HO}^{2}$ and the analytical expression of the GPhC EFSs, we estimated the output beam position as a function of wavelength. The results are plotted in Figure 4 and superimposed to the experimental data. A quantitative agreement between HypSNOM experimental results and 3D-PWE-assisted HO propagation is clearly obtained. This whole consistency shows that near-field measurements can successfully probe electromagnetic phenomena in artificial optical materials. It also proves that modeling the light propagation using the local band structure in the approximation of a slow variation of opto-geometrical parameters inherent to the HO formalism is valid here.

To conclude, hyperspectral near-field optical observations of the mirage effect in a graded photonic crystal area are reported. The employed method allows a direct experimental investigation of the mirage effect and its dispersion over the $1200 \mathrm{~nm}-1650 \mathrm{~nm}$ spectral range, giving insight into the physics of dispersive electromagnetic phenomena in these unusual artificial optical materials. A quantitative agreement is obtained between experimental and simulation results in the considered $90^{\circ}$-light bending structure, validating the whole modelling/experiment approach consistency. The reported results illustrate the capabilities of the hyperspectral near-field imaging technique to quantify the dispersive phenomena in artificial materials. They also open promising perspectives for light manipulation using graded photonic crystals.

The authors acknowledge the Burgundy Regional Council from France for financial support through the PHOTCOM project.

${ }^{1}$ A. Boltasseva and V. M. Shalaev, Metamaterials 2, 1 (2008).

${ }^{2}$ P. S. J. Russel and T. A. Birks, IEEE J. Lightwave Technol. 17, 19821988 (1999).

${ }^{3}$ E. Centeno and D. Cassagne, Opt. Lett. 30, 2278-2280 (2005).

${ }^{4}$ E. Centeno, D. Cassagne, and J. P. Albert, Phys. Rev. B 73, 235119 (2006).

${ }^{5}$ L. H. Gabrielli and M. Lipson, Opt. Express 19, 20122 (2011).

${ }^{6}$ T. Zentgraf, J. Valentine, N. Tapia, J. Li, and X. Zhang, Adv. Mater. 22, 2561 (2010).

${ }^{7}$ L. H. Gabrielli, J. Cardenas, C. B. Poitras, and M. Lipson, Nat. Photonics 3, 461 (2009).

${ }^{8}$ D. A. Genov, S. Zhang, and X. Zhang, Nat. Phys. 5, 687 (2009). 
${ }^{9}$ D. W. Pohl, W. Denk, and M. Lanz, Appl. Phys. Lett. 44, 651 (1984).

${ }^{10}$ M. Burresi, D. Diessel, D. van Oosten, S. Linden, M. Wegener, and L. Kuipers, Nano Lett. 10, 2480-2483 (2010).

${ }^{11}$ L. Lalouat, B. Cluzel, C. Dumas, L. Salomon, and F. de Fornel, Phys. Rev. B 83, 115326 (2011).

${ }^{12}$ L. Aigouy, P. Lalanne, J. P. Hugonin, G. Julié, V. Mathet, and M. Mortier, Phys. Rev. Lett. 98, 153902 (2007).

${ }^{13}$ M. Burresi, D. van Oosten, T. Kampfrath, H. Schoenmaker, R. Heideman, A. Leinse, and L. Kuipers, Science 326, 550-553 (2009).

${ }^{14}$ M. L. M. Balistreri, J. P. Korterik, L. Kuipers, and N. F. van Hulst, Phys. Rev. Lett. 85, 294 (2000).

${ }^{15}$ M. L. M. Balisteri, H. Gersen, J. P. Korterik, L. Kuipers, and N. F. van Hulst, Science 294, 1080-1082 (2001).
${ }^{16}$ R. J. P. Engelen, Y. Sugimoto, H. Gersen, N. Ikeda, K. Asakawa, and L. Kuipers, Nat. Phys. 3, 401-405 (2007).

${ }^{17}$ J. S. Bouillard, S. Vilain, W. Dickson, and A. V. Zayats, Opt. Express 18, 16513-16519 (2010).

${ }^{18}$ E. Akmansoy, E. Centeno, K. Vynck, D. Cassagne, and J. M. Lourtioz, Appl. Phys. Lett. 92, 133501 (2008).

${ }^{19}$ E. Cassan, K.-V. Do, C. Caer, D. Marris-Morini, and L. Vivien, IEEE J. Lightwave Technol. 29, 1937 (2011).

${ }^{20}$ K.-V. Do, X. L. Roux, D. Marris-Morini, L. Vivien, and E. Cassan, Opt. Express 20, 4476 (2012).

${ }^{21}$ I. Newton, Newton's Optics (Smith \& Walford, London, 1704).

${ }^{22}$ J. Dellinger, D. Bernier, B. Cluzel, X. L. Roux, A. Lupu, F. de Fornel, and E. Cassan, Opt. Lett. 36, 1074-1076 (2011). 